\title{
STUDY OF THE CORRELATION BETWEEN THE CONTENT OF NANOOBJECTS AND SURFACE FEATURES \\ Natalia Kamanina ${ }^{1}$
}

\begin{abstract}
It is well known that at the present time two basic aspects of a material's nano-objects sensitization should be considered. The first aspect relates to the change of the material's basic physical-chemical properties when the concentration of the nanoobjects in the material's body is varied. The second aspect is in regard to the modification of surface properties on the content of the nanoobjects in the material's body. In the current paper the correlation between the concentration of the nanoobjects and wetting phenomena of the material's surface is considered. Different experimental instruments and supporting models are presented with good coincidence.
\end{abstract}

UDC Classification: 532.783; 535.33; 539.6; 539.8; 538.95, DOI: https://doi.org/10.12955/pns.v2.152

Keywords: Organic materials, surface relief, sensitization, fullerenes and nanotubes content, polyvinyl alcohol, polyimide, wetting angle

\section{Introduction}

Currently, more and more nanotechnological aspects are considered, both from a fundamental point of view for expanding the material science knowledge, and from a practical point of view, due to the widespread use of the nanomaterials and devices based on them in general optoelectronics and biomedicine. Different nanoparticles (NPs) are used for these aims. Fullerenes (Krätschmer, 1990a; Krätschmer, 1990b; Liu, 1994; Couris, 1995; Brabec, 1999; Belousov, 1999; Kamanina, 2002), carbon nanotubes (CNTs) (Robertson, 2004; Namilae, 2004; Ould-Moussa, 2013), quantum dots (Liu, 2003; Babaev, 2019), shungites (Rozhkova, 2007; Sheka, 2014), and reduced graphene oxides (Sobon, 2012; Abbaspour, 2017) are studied and applied to dope organic materials in order to reveal a change in their basic physical-chemical parameters.

Recently an interesting phenomenon has been predicted and found when the core (skeleton) of the nanoobjects introduced in the organic material's body influences the surface properties. The Lotus effect can be observed, the realization of the liquid crystal molecules orientation on the established surface relief has been shown (Kamanina, 2019a; Kamanina, 2019b), and an additional optical limiting mechanism can be found (Kamanina, 2020a).

In the current paper, another aspect of the surface modification due to the content of the nanoobjects varying in the material's body is considered. The correlation between the content of the nanoobjects inside the organic matrixes based on the polyvinyl alcohol and polyimide and their surface features has been established, shown, and explained.

\section{Experimental conditions, materials and results}

As the model material, polyvinyl alcohol (PVA) (structure, type \#182480-500MG (in catalog Alfa Aesar and Aldrich) with the molecular mass of 100000 a.u. and polyimide (PI) structure obtained from L.Ya.Karpov Research Institute of Physics and Chemistry, Moscow, Russia) was used. PVA water solution was made with the content of the dry PVA in water of $8.0 \mathrm{wt} . \%$. As the nanoobjects the fullerenes $\mathrm{C}_{70}$ powder with a purity of $97 \%$ and single wall carbon nanotubes (SWCNTs), type \#704121, with their diameter placed in the range of 0.7-1.1 nm, purchased from Alfa Aesar and Aldrich Co., was applied. The content of the CNTs in the water solution of the PVA matrix was varied from 0.1 to $10 \mathrm{wt} \%$. The content of the fullerenes in the polyimide media were varied from 0.1 up to 1 wt.\%. The thin polyimide films were prepared from 3-6.5\% solutions in the tetrachloroethane solvent and then the compounds were poured by centrifugation onto the glass substrates.

OCA 15EC instrument, purchased from LabTech Co. (Saint-Petersburg-Moscow, Russia), was used in order to establish the change of the wetting angle at the modified polymer surfaces, when the content of the nanoobjects in the model material's body was varied.

It should be noted that the wetting angle increases due to an increase in the nanoobjects concentration, which directly changed the surface relief. In reality this is because of the nanoobjects' hexagonal and

\footnotetext{
${ }^{1}$ Vavilov State Optical Institute, St.- Petersburg, Russia; St.-Petersburg Electrotechnical University ("LETI"), St.-Petersburg, Russia; Kurchatov Institute - Nuclear Physics Institute, St.-Petersburg-Gatchina, nvkamanina@mail.ru
} 
pentagonal core that changed the roughness of the surface. The data shown in Fig.1 indicate some results of wetting angle increase via content of the CNTs change in the PVA matrix. This figure reveals the dependence of the wetting angle of the water drops at the surface of thin-film PVA matrix with CNTs concentrations varying of $0.1 ; 0.5 ; 1.0 ; 5.0$ and 10.0 wt. $\%$.

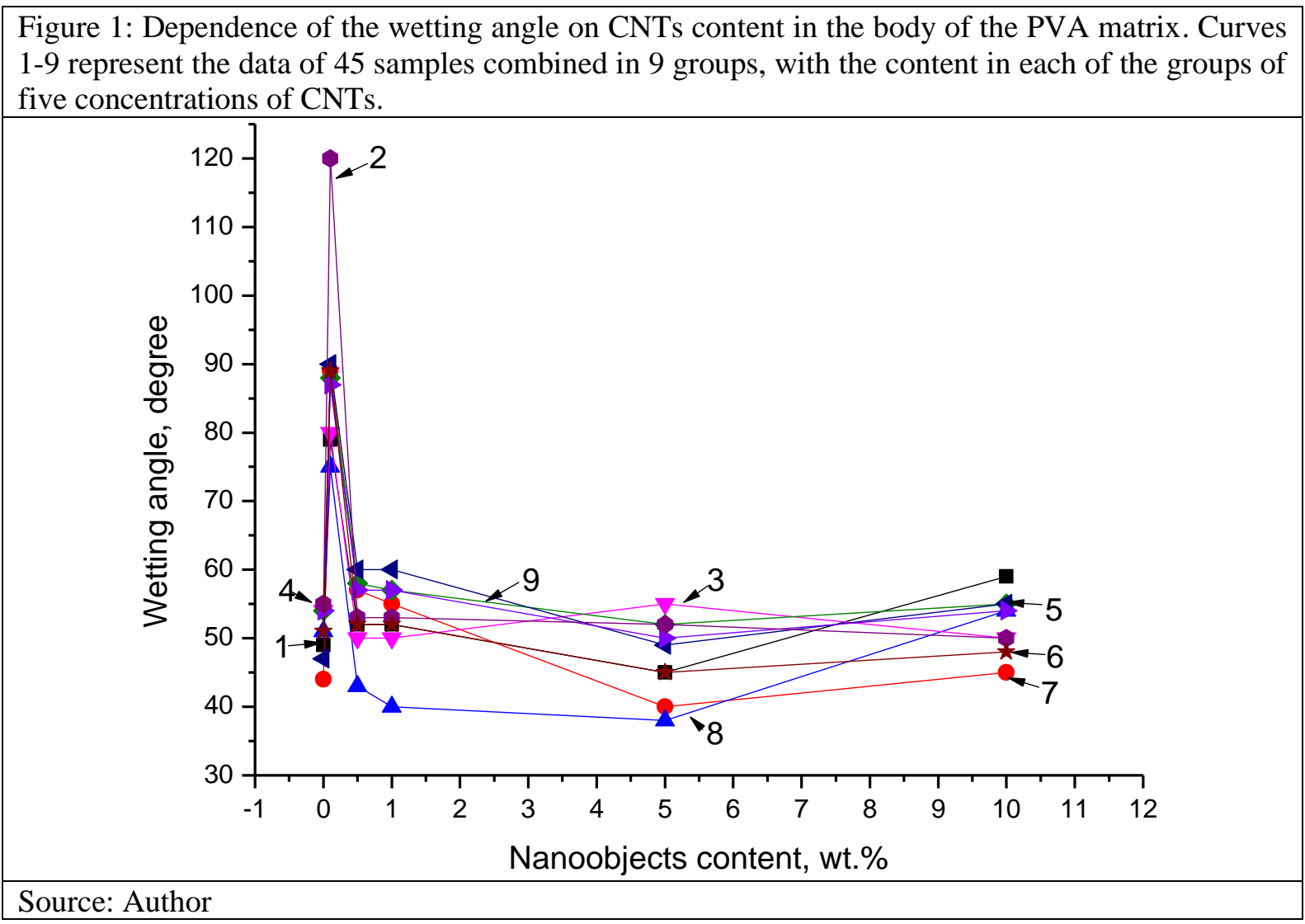

Analyzing the data obtained from Fig.1 one can testify that there is a threshold value of the slope angle after which its stationary value is set. For the PVA matrix doped with the 0.1 and $0.5 \mathrm{wt} . \%$ of the CNTs the maximum wetting angle can be found. At high concentrations of nanotubes, most likely, the angle saves the stationary level, perhaps, due to the interweaving of the nanotubes themselves in the PVA matrix and their inhomogeneous distribution.

Moreover, this saturation level of the wetting angle on the nanoobjects content can be probably explained by the fact that the PVA polymer chains themselves can enter into the nanotubes, apparently causing a change in the distribution of these chains over the volume of the PVA material. Some quantum chemical modeling supported this fact has been made before (Kamanina, 2011); an additional picture, which can present this process is shown in Fig.2. It should be remarked that the quantumchemical simulations of the modified structure and the penetration effect have been performed using GULP and TBDFT (Das, 2009) programs. Using the molecular dynamics method for a simple system of an SWCNT on a PVA matrix, the simulation has employed an SWCNT fragment with a diameter of $1 \mathrm{~nm}$ and a length of 4-5 nm on a $4 \mathrm{~nm}$ thick PVA film with an area of $6 \times 6 \mathrm{~nm}^{2}$. The nanotube has been incorporated into the matrix to a depth of $\sim 2 \mathrm{~nm}$. In Figure 2 is shown the introduction of the PVA into CNTs.

Returning to Fig.1, it should be remarked that the same evidence of the wetting angle change via the content of the nanoobjects varying has been obtained for the PVA matrix structured with the "Taunit" NPs shown in the paper by Kamanina (2020b). The maximum level of the wetting angle and its output to a stationary value were also determined by varying the concentration of nanoobjects in the volume of the PVA matrix.

Thus, considering some practical application, one can conclude that this surface relief can be useful for the display technique because it can be easy modified, for example, for the liquid crystal molecules orientation from the orthogonal to planar position (and back) and can be proposed, for comparison, in 
the TN- and MWVA-display technology. This effect can also be considered in polarizing optics in order to vary the transmittance of the parallel and orthogonal light components.

Figure 2: Model representation of the process of how the PVA polymer chains penetrated into the carbon nanotubes.

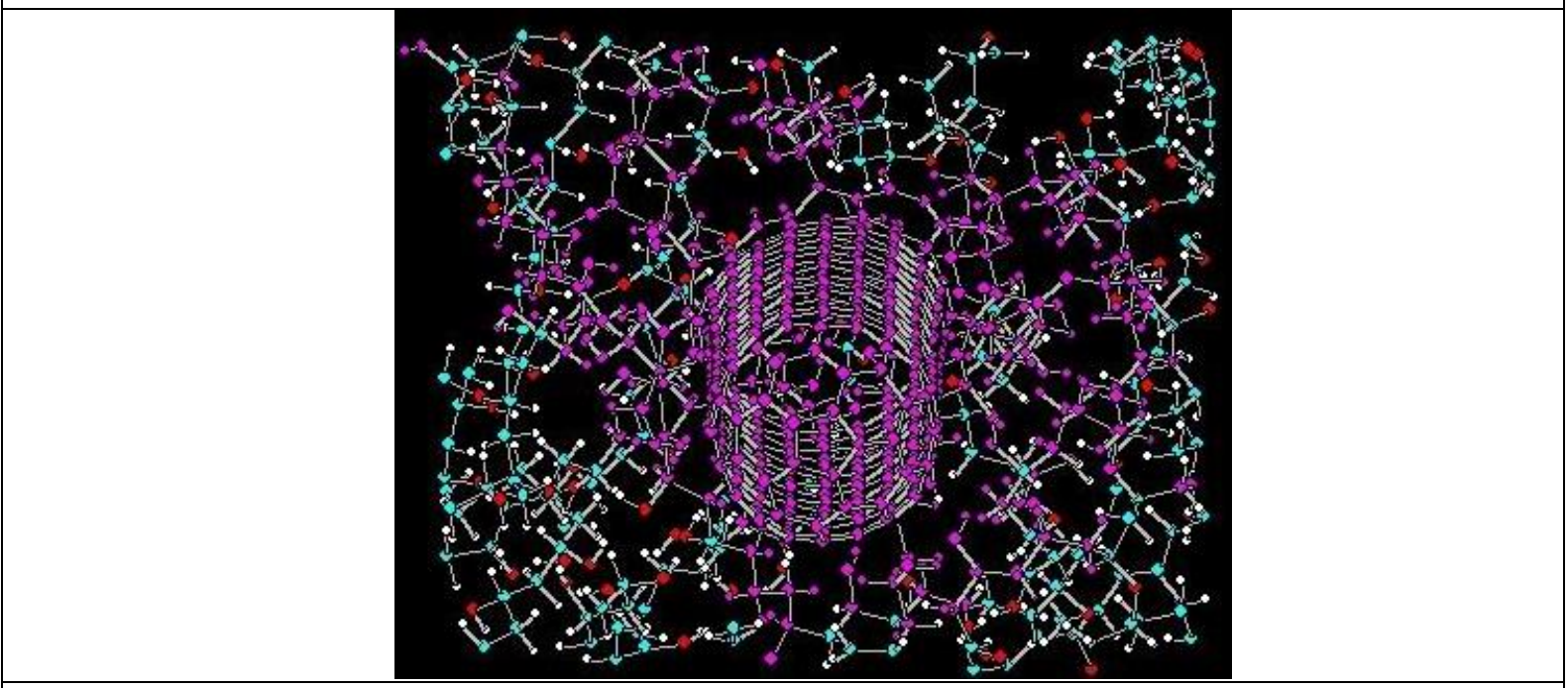

Source: Author

The data shown in Table 1 indicate some results of wetting angle increase via content of the fullerene $\mathrm{C}_{70}$ change in the polyimide matrix. This data reveals the dependence of the wetting angle of the water drops at the surface of thin-film PI matrix with the fullerene $\mathrm{C}_{70}$ concentrations varying of $0.1 ; 0.2$; 0.5 ; and 1.0 wt. $\%$.

Table 1: Comparative results of the wetting angle change with an increase in the fullerene $\mathrm{C}_{70}$ content in the model polyimide matrix.

\begin{tabular}{|l|c|c|c|c|c|c|c|}
\hline Structure & $\begin{array}{c}\text { Nanoobjects } \\
\text { content, wt.\% }\end{array}$ & \multicolumn{7}{|c|}{ Wetting angle, degree } \\
\hline PI pure & 0 & 60 & 61 & 60 & 60 & 63 & 61 \\
\hline PI+0,1wt.\%C 70 & 0.1 & 70 & 70 & 71 & 70 & 70 & 71 \\
\hline PI+0,2wt.\%C 70 & 0.2 & 74 & 75 & 75 & 74 & 73 & 75 \\
\hline PI+0,5wt.\%C & 0.5 & 77 & 78 & 78 & 77 & 79 & 77 \\
\hline PI+1,0 wt.\%C & 1 & 93 & 90 & 93 & 89 & 90 & 89 \\
\hline Source: author & &
\end{tabular}

One can testify that other evidence can be registered when the organic matrix has been doped by an intermolecular strong acceptor such as the fullerene $\mathrm{C}_{70}$. A clear and consistent increase in the wetting angle of the water drops is observed on the surface of the sensitized polyimide with an increase in the concentration of fullerene $\mathrm{C}_{70}$.

Due to the fact that fullerenes are actively involved in the creation of an intermolecular charge transfer complex with a triphenylamine donor fragment of a polyimide molecule (Kamanina, 2012) the uniform distribution of fullerenes in the polymer matrix is observed. Really, it should be taken into account that, for example, the monomeric links of pure polyimides are intramolecular donor-acceptor (D-A) complexes with the charge transfer between the donor and acceptor molecular fragments, which can be changed under the condition of intermolecular acceptor introduction. Polyimides consist of acceptor diimide fragments with the electron affinity energy of $1.12-1.46 \mathrm{eV}$ and of donor triphenylamine fragments with the ionization potential of $6.5-9.2 \mathrm{eV}$. Low ionization potential allowed the $\mathrm{D}-\mathrm{A}$ complexes to be formed between monomeric links of the polyimide and incorporated acceptor molecules during both structural-chemical and injection sensitizations. Thus, according to the data in Table 1, one can observe an increase of the wetting angle without the need for saturation, possibly because of the influence of the intermolecular $\mathrm{D}-\mathrm{A}$ on the nanoobjects' regular distribution. It is shown in the growth of the wetting angle as an increase in the concentration of nanoobjects. 


\section{Conclusion}

To summarize the obtained results and proposed idea, one can present the following interesting aspects, which can extend our knowledge in the area of material science and which can be useful for future practical applications.

For each organic matrix, there is a certain concentration range of the dopant (sensitizer), at which there is a variation in the configuration of the surface relief. The surface configuration can be tested via the wetting angle of the water molecules drop testing. For organic materials without the initial donoracceptor interaction (for example, the PVA matrix), there is an original angle of inclination of the water molecules on the surface, which is directly related to the concentration of the introduced dopant in the organic material's body. It is worth noting that for the organic materials with an initial donoracceptor interaction and for the materials without such a property, the tendency to change the angle of inclination depending on the concentration of nanoobjects inside the polymers will be different. Of course, such studies should be continued for greater confidence in the observed effect, taking into account its testing in other polymer matrices. The dependence of the angle of the water molecule drops on the surface of the thin organic films helps to forecast the prospect of their use in LC display technology, in the modulation technique, and in the explanation of the additional optical limiting radiation effect as well. It can also be useful for general optoelectronics and biomedicine.

\section{Acknowledges}

The author would like to thank her colleagues from the Lab for Photophysics of media with the nanoobjects (Vavilov State Optical Institute, Saint-Petersburg, Russia) for the helpful discussion at the Lab seminars. Moreover, the author thanks Prof. L. Chernozatonslii (Moscow, Russia) for the help with the quantum-chemical simulation and Dr. V.I. Berendyaev for the polyimide structure presenting. Some part of the obtained results has been discussed at the seminar in the Vincha Institute (Belgrade, Serbia, 2017), in the Nuclear Research Institute (Gatchina, Saint-Petersburg, Russia, 2017, 2020) and at the Prague CBU Research Institute (Prague, Czech Republic, 2021).

\section{References}

Krätschmer, W., Fostiropoulos, K., Huffman, D.R. (1990a).The infrared and ultraviolet absorption spectra of laboratoryproduced carbon dust: evidence for the presence of the C60 molecule. Chem. Phys. Lett., 170(2-3), 167-170.

Krätschmer, W., Lamb, L.D., Fostiropoulos, K., Huffman, D.R. (1990b). Solid C60: a new form of carbon. Nature, 347,354 358.

Liu, Huimin, Taheri, B., Weiyi, Jia. (1994).Anomalous optical response of $\mathrm{C}_{60}$ and $\mathrm{C}_{70}$ in toluene. Phys. Rev. B, 49(15), 10166-10169.

Couris, S., Koudoumas, E., Ruth, A. A., Leach, S. (1995). Concentration and wavelength dependence of the effective thirdorder susceptibility and optical limiting of $\mathrm{C}_{60}$ in toluene solution. J. Phys. B: At. Mol. Opt. Phys., 28, 4537-4554.

Brabec, C.J., Padinger, F., Sariciftci, N.S., Hummelen, J.C. (1999). Photovoltaic properties of conjugated polymer/ methanofullerene composites embedded in a polystyrene matrix. Journal of Applied Physics, 85(9), 6866-6872

Belousov, V.P., Belousiva, I.M., Gavronskaya, E.A., Grigor'ev V.A., Danilov, O.B., Kalintsev, A.G., Krasnopolskij V.E., Smirnov, V.A., Sosnov E.N. (1999). About optical limiting mechanisms in the fullerene-containing media. Optics and Spectroscopy, 87(5), 845-852.

Kamanina, N.V. (2002). Mechanisms of optical limiting in $\pi$-conjugated organic system: fullerene-doped polyimide. Synthetic Metals, 127(1-3), 121-128. DOI: 10.1016/S0379-6779(01)00598-7.

Robertson, J. (2004). Realistic applications of CNTs. Materials Today. 7(10), 46-52.

Namilae, S., Chandra, N., Shet, C. (2004). Mechanical behavior of functionalized nanotubes. Chemical Physics Letters, 387(4-6), 247-252.

Ould-Moussa, N., Blanc, Ch., Zamora-Ledezma, C., Lavrentovich, O.D., Smalyukh, I.I., Islam, M.F., Yodh, A.G., Maugey, M., Poulin, P., Anglaret E., M. Nobili. (2013). Dispersion and orientation of single-walled carbon nanotubes in a chromonic liquid crystal. Liquid Crystals, 40(12), 1628-1635, DOI:10.1080/02678292.2013.772254.

Liu, S.H., Qian, X.F., Yin, J., Xi, H.A., Huang, Z.H., Zhu, Z.K. (2003). Fabrication of CdS nanocrystals embedded in copolymer matrix by an in situ simultaneous copolymerization-sulfidation technique. Materials Science and Engineering B, 98(2), 99-103. doi:10.1016/S0921-5107(02)00353-7

Babaev, A.A., Parfenov, P.S., Onishchuk, D.A., Dubavik, A., Cherevkov, S.A., Rybin, A.V., Baranov, M.A., Baranov, A.V. Litvin, A.P., Fedorov, A.V. (2019). Functionalized rGO Interlayers Improve the Fill Factor and Current Density in PbS QDsBased Solar Cells. Materials (Basel, Switzerland), 12(14), 4221, 10 pages. doi:10.3390/ma12244221

Rozhkova, N.N., Gribanov, A.V., Khodorkovskii, M.A. (2007). Water mediated modification of structure and physical chemical properties of nanocarbons. Diamond \& Related Materials, 16(12), 2104-2108. 
Sheka, E.F., Rozhkova, N.N. (2014). Shungite as the natural pantry of nanoscale reduced graphene oxide. International Journal of Smart and Nano Materials, 5(1), 1-16, doi: 10.1080/19475411.2014.885913

Sobon, G., Sotor, J., Jagiello, J., Kozinski, R., Zdrojek, M., Holdynski, M., Paletko, P., Boguslawski, J., Lipinska, L, and Abramski, K.M. (2012). Graphene Oxide vs. Reduced Graphene Oxide as saturable absorbers for Er-doped passively modelocked fiber laser. Optics Express, 20(17), 19463-19473.

Abbaspour, M., Pourabbas, B., Azimi, M., Abdeali, G., Asgari, A. (2017). Solid-state supercapacitor based on breath figured polymethylmethacrylate deposited by graphene: the effect of electrode surface. J Mater Sci: Mater Electron, 28, 1412114130, doi 10.1007/s10854-017-7265-z

Kamanina, Natalia V. (2019a). Nanoparticles doping influence on the organics surface relief. Journal of Molecular Liquids, $283,65-68$.

Kamanina, Natalia. (2019b). Liquid crystal materials orientation using new approach. Proceed. of CBU International conference on innovations in science and education, March 20-22, Prague, Czech Republic, 933-937, doi: 10.12955/cbup.v7.147

Kamanina, Natalia. (2020a). Some aspects of the materials' optical limiting features. Proceedings of CBU in Natural Sciences and ICT, 1, 33-36.2020, Prague, Czech Republic, doi: https://doi.org/10.12955/pns.v1.118

Kamanina, N.V., Likhomanova, S.V., Vasilyev, P.Ya., Studeonov, V.I., Chernozatonskii, L.A., Vaganov, V.E., Mishakov, I. V. (2011). Surface Properties of Thin-Film Polarizers Modified by Carbon Nanostructures. Tech. Phys.Lett. 37(12), 11651167.

Ramamurty, U., Rao C.N.R. (2009). Nano-indentation studies on polymer matrix composites reinforced by few-layer graphene. Nanotechnology 20(12), 125705, 5 pages. doi: 10.1088/0957-4484/20/12/125705

Kamanina, N.V., Studeonov, V.I., Tkachev, A.G. (2020b). Thin-film light polarizers: media bulk structuring and surface modifying. Liq. Cryst. and their Appl. 20 (4), 78-83.

Kamanina, N.V., Serov, S.V., Shurpo, N.A., Likhomanova, S.V., Timonin, D.N., Kuzhakov, P.V., Rozhkova, N.N., Kityk, I.V., Plucinski, K. J., Uskokovic, D. P. (2012). Polyimide-fullerene nanostructured materials for nonlinear optics and solar energy applications. J Mater Sci: Mater Electron, 23(8), 1538-1542, doi 10.1007/s10854-012-0625-9. 